\title{
LS-SVM approximate solution to linear time varying descriptor systems
}

\author{
S. Mehrkanoon ${ }^{1}$, J.A.K. Suykens \\ KU Leuven, ESAT-SCD, \\ Kasteelpark Arenberg 10, B-3001 Leuven (Heverlee), Belgium.
}

\begin{abstract}
This paper discusses a numerical method based on Least Squares Support Vector Machines (LS-SVMs) for solving linear time varying initial and boundary value problems in Differential Algebraic Equations (DAEs). The method generates a closed form (model-based) approximate solution. The results of numerical experiments on different systems with index from 0 to 3 , are presented and compared with analytic solutions to confirm the validity and applicability of the proposed method.
\end{abstract}

Key words: Least squares support vector machines, descriptor systems, closed form approximate solution

\section{Introduction}

Differential algebraic equations (DAEs) arise frequently in numerous applications including mathematical modelling, circuit and control theory [6], chemistry [10, 22], fluid dynamic [15] and computer-aided design. DAEs have been known under a variety of names, depending on the area of application for instance they are also called descriptor, implicit or singular systems. The most general form of DAE is given by

$$
F(\dot{x}, x, t)=0
$$

where $\frac{\partial F}{\partial \dot{x}}$ may be singular. The rank and structure of this Jacobian matrix depends, in general, on the solution $x(t)$. DAEs are characterized by their index. In [7] the index of (1) is defined as the minimum number of differentiations of the system which would be required to solve for $\dot{x}$ uniquely in terms of $x$ and $t$. The index of DAEs is a measure of the degree of singularity of the system and it is widely considered as an indication of certain difficulties for numerical methods. We note that DAEs with an index greater than 1 are often referred to as higher-index DAEs and that the index of an ODE is zero. See $[2,8]$ for a detailed discussion of the index of a DAE. The important special case of (1) is semi-explicit DAE or an ODE with constraints i.e.

$$
\begin{aligned}
& \dot{x}=f(x, y, t) \\
& 0=g(x, y, t) .
\end{aligned}
$$

The index is 1 if $\frac{\partial g}{\partial y}$ is nonsingular. $x$ and $y$ are considered as differential and algebraic variables respectively. Analytic solutions for these problems are not generally available and hence numerical methods must be applied. Some numerical methods

\footnotetext{
${ }^{1}$ Corresponding author. E-mail addresses: \{Siamak.Mehrkanoon, Johan.Suykens\}@esat.kuleuven.be
}

have been developed for solving DAEs using backward differentiation formulas (BDF) [1, 7, 11, 2, 3] or implicit RungeKutta (IRK) methods [7, 3, 4, 19]. These methods are only applicable to low-index problems and often require that the problem to have special structure. Furthermore, these approaches approximate the solutions at discrete points only (discrete solution) and some interpolation techniques is needed in order to get continuous solution. Thereby, recently attempts have been made to develop methods that produce a closed form approximate solution. Awawdeh et al. [5] applied Homotopy analysis method to systems of DAEs. The authors in [12] used Padé approximation method to estimate the solution of singular systems with index-2.

In general, the higher the index, the greater numerical difficulty one is going to encounter when solving differential algebraic equations numerically and an alternative treatment is the use of index reduction techniques which are based on repeated differentiation of the constraints until a low-index problem (an index 1 DAE or ODE) is obtained. There are several reasons to consider differential algebraic equations (2) directly, rather than convert it to system of ODEs [7, 25]. Therefore designing direct methods that do not require a reformulation (e.g. index reduction) of DAEs will not only speed up the solution, also the system structure (e.g. the modelling changes and parameter variations) can be more readily explored.

It is known that the singular system can have instantaneous jumps due to inconsistent initial conditions. Several approaches for consistent initialization of DAEs have been studied in the literature and in general they fall into two categories: rigorous initialization and the direct initialization method (we refer the reader to [21] and references therein for more details). Within the scope of this paper we assume that consistent initial conditions are available.

In [16], solving ordinary differential equations using Least Squares Support Vector Machines has been discussed. In this paper the method developed in [16] is extended to approximate 
the solution of linear time varying initial and boundary value problems in differential algebraic equations.

This paper is organized as follows. In Section 2, a brief review about least squares support vector regression problems together with preliminary definitions are given. In Section 3, we formulate our least squares support vector machines method to the solution of linear time-varying initial value problem (IVPs) in DAEs. Section 4, is devoted to the formulation of the method for boundary value problems (BVPs) in DAEs. The model selection of the proposed method is discussed in Section 5. In Section 6, numerical experiments are tested to demonstrate the viability of the proposed method.

\section{LS-SVM regression and preliminary definitions}

Let us consider a given training set $\left\{x_{i}, y_{i}\right\}_{i=1}^{N}$ with input data $x_{i} \in \mathbb{R}$ and output data $y_{i} \in \mathbb{R}$. The goal in regression is to estimate a model of the form $y(x)=w^{T} \varphi(x)+b$.

The primal LS-SVM model for regression can be written as follows [23]

$$
\begin{array}{ll}
\underset{w, b, e}{\operatorname{minimize}} & \frac{1}{2} w^{T} w+\frac{\gamma}{2} e^{T} e \\
\text { subject to } & y_{i}=w^{T} \varphi\left(x_{i}\right)+b+e_{i}, \quad i=1, \ldots, N
\end{array}
$$

where $\gamma \in \mathbb{R}^{+}, b \in \mathbb{R}, w \in \mathbb{R}^{h} . \varphi(\cdot): \mathbb{R} \rightarrow \mathbb{R}^{h}$ is the feature map and $h$ is the dimension of the feature space. The dual solution is then given by

$$
\left[\begin{array}{c|c}
\Omega+I_{N} / \gamma & 1_{N} \\
\hline 1_{N}^{T} & 0
\end{array}\right]\left[\begin{array}{c}
\alpha \\
\hline b
\end{array}\right]=\left[\begin{array}{c}
y \\
\hline 0
\end{array}\right]
$$

where $\Omega_{i j}=K\left(x_{i}, x_{j}\right)=\varphi\left(x_{i}\right)^{T} \varphi\left(x_{j}\right)$ is the $(i, j)$-th entry of the positive definite kernel matrix. $1_{N}=[1 ; \ldots ; 1] \in \mathbb{R}^{N}$, $\alpha=\left[\alpha_{1} ; \ldots ; \alpha_{N}\right], y=\left[y_{1} ; \ldots ; y_{N}\right]$ and $I_{N}$ is the identity matrix. When we deal with differential equations, the target values $y_{i}$ are no longer directly available, so the regression approach will fail. Nevertheless we can incorporate the underlying differential equation in the learning process to find an approximation for the solution.

First we need to define the derivative of the kernel function. Making use of Mercer's Theorem [24], derivatives of the feature map can be written in terms of derivatives of the kernel function. Let us define the following differential operator which will be used in subsequent sections

$$
\nabla_{n}^{m} \equiv \frac{\partial^{n+m}}{\partial x^{n} \partial y^{m}}
$$

If $\varphi(x)^{T} \varphi(y)=K(x, y)$, then one can show that

$\left[\varphi^{(n)}(x)\right]^{T} \varphi^{(m)}(y)=\nabla_{n}^{m}\left[\varphi(x)^{T} \varphi(y)\right]=\nabla_{n}^{m}[K(x, y)]=\frac{\partial^{n+m} K(x, y)}{\partial x^{n} \partial y^{m}}$.

Using formula (5), it is possible to express all derivatives of the feature map in terms of the kernel function itself (provided that the kernel function is sufficiently differentiable). For instance if we choose the RBF kernel, $K(x, y)=\exp \left(-\frac{(x-y)^{2}}{\sigma^{2}}\right)$, then the following relations hold,

$$
\begin{aligned}
& \nabla_{0}^{0}[K(x, y)]=K(x, y), \\
& \nabla_{1}^{0}[K(x, y)]=\frac{\partial\left(\varphi(x)^{T} \varphi(y)\right)}{\partial x}=\varphi^{(1)}(x)^{T} \varphi(y)=-\frac{2(x-y)}{\sigma^{2}} K(x, y), \\
& \nabla_{0}^{1}[K(x, y)]=\frac{\partial\left(\varphi(x)^{T} \varphi(y)\right)}{\partial y}=\varphi(x)^{T} \varphi^{(1)}(y)=\frac{2(x-y)}{\sigma^{2}} K(x, y) .
\end{aligned}
$$

The superscript ${ }^{(1)}$ stands for the derivative operator.

\section{Formulation of the method for IVPs in DAEs}

Consider linear time varying initial value problem in DAEs of the following from

$$
\begin{aligned}
& Z(t) \dot{X}(t)=A(t) X(t)+B(t) u(t), t \in\left[t_{i n}, t_{f}\right], \\
& X\left(t_{\text {in }}\right)=X_{0},
\end{aligned}
$$

where $Z(t)=\left[z_{i j}(t)\right], A(t)=\left[a_{i j}(t)\right] \in \mathbb{R}^{m \times m}$ and $B(t) \in \mathbb{R}^{m \times r}$. The state vector $X=\left[x_{1}, \ldots, x_{m}\right]^{T} \in \mathbb{R}^{m}$, the input vector $u \in \mathbb{R}^{r}$ and $\dot{X}(t)=\frac{d X}{d t} . Z(t)$ may be singular on $\left[t_{i n}, t_{f}\right]$ with variable rank and the DAE may have an index that is larger than one. When $Z$ is nonsingular, equation (6) can be converted to equivalent explicit ODE system. In addition we assume that $Z(t)$, $A(t)$ and $B(t) u(t)$ are sufficiently smooth and the DAE (6) is solvable. (see Definition 2.1 in [9] and references therein for a more detailed discussion about solvability)

Let us assume that a general approximate solution to $i$-th equation of (6) is of the form of $\hat{x}_{i}(t)=w_{i}^{T} \varphi(t)+b_{i}$, where $w_{i}$ and $b_{i}$ are parameters of the model that have to be determined. To obtain the optimal value of these parameters, collocation methods can be used [14] which assume a discretization of the interval $\left[t_{i n}, t_{f}\right]$ into a set of collocation points $\Upsilon=\left\{t_{\text {in }}=t_{1}<t_{2}<\ldots<t_{N}=t_{f}\right\}$. Therefore the adjustable parameters $w_{i}$ and $b_{i}$, for $i=1, \ldots, m$, are to be found by solving the following optimization problem:

$$
\begin{array}{ll}
\underset{\hat{X}}{\operatorname{minimize}} & \frac{1}{2} \sum_{i=1}^{N}\left[\left(Z \hat{X}^{\prime}-A \hat{X}-B u\right)\left(t_{i}\right)\right]^{2} \\
\text { subject to } & \hat{X}\left(t_{i n}\right)=X_{0} .
\end{array}
$$

where $N$ is the number of collocation points (which is equal to the number of training points) used to undertake the learning process. For simplicity let us assume that $X_{0}=\left[p_{1} ; \ldots ; p_{m}\right]$ and $g(t)=B(t) u(t)$. In what follows we formulate the optimization problem in the LS-SVM framework for solving linear time varying differential algebraic equations. For notational convenience let us list the following notations which are used in the 
following sections,

$$
\begin{aligned}
& {\left[\nabla_{n}^{m} K\right](t, s)=\left.\left[\nabla_{n}^{m} K(x, y)\right]\right|_{x=t, y=s},} \\
& \Omega_{n}^{m}(i, j)=\left.\nabla_{n}^{m}[K(x, y)]\right|_{x=t_{i}, y=t_{j}}=\left.\frac{\partial^{n+m} K(x, y)}{\partial x^{n} \partial y^{m}}\right|_{x=t_{i}, y=t_{j}}, \\
& \Omega_{0}^{0}(i, j)=\left.\nabla_{0}^{0}[K(x, y)]\right|_{x=t_{i}, y=t_{j}}=K\left(t_{i}, t_{j}\right) .
\end{aligned}
$$

Where $\Omega_{n}^{m}(i, j)$ denotes the $(i, j)$-th entry of matrix $\Omega_{n}^{m}$. The Matlab notation $M(k: l, m: n)$ is used for selecting a submatrix of matrix $M$ consisting of rows $k$ to $l$ and columns $m$ to $n$. $M(i$, : ) denotes the $i$-th row of matrix $M . M(:, j)$ denotes the $j$-th column of matrix $M$.

Remark 1. In order to keep the notations as simple as possible, we utilized the same feature map $\varphi(t)$ for all the states, i.e. $\hat{x}_{i}(t)=w_{i}^{T} \varphi(t)+b_{i}$. Nevertheless it is possible to use different mapping functions, as long as the corresponding kernel functions satisfy the Mercer's Theorem [24], and then one has more hyper parameters to tune.

\subsection{Singular ODE System}

Let us consider DAEs (6). The approximate solution, $\hat{x}_{i}(t)=$ $w_{i}^{T} \varphi(t)+b_{i}$, for $i=1, \ldots, m$ is then obtained by solving the following optimization problem

$$
\begin{aligned}
\underset{w_{i}, b_{i}, e_{\ell}^{i}}{\operatorname{minimize}} & \frac{1}{2} \sum_{\ell=1}^{m} w_{\ell}^{T} w_{\ell}+\frac{\gamma}{2} \sum_{\ell=1}^{m} e_{\ell}^{T} e_{\ell} \\
\text { subject to } & {\left[\begin{array}{ccc}
z_{11}(t) & \cdots & z_{1 m}(t) \\
\vdots & \ddots & \vdots \\
z_{m 1}(t) & \cdots & z_{m m}(t)
\end{array}\right]\left[\begin{array}{c}
w_{1}^{T} \varphi^{\prime}\left(t_{i}\right) \\
\vdots \\
w_{m}^{T} \varphi^{\prime}\left(t_{i}\right)
\end{array}\right]=} \\
& {\left[\begin{array}{ccc}
a_{11}(t) & \cdots & a_{1 m}(t) \\
\vdots & \ddots & \vdots \\
a_{m 1}(t) & \cdots & a_{m m}(t)
\end{array}\right]\left[\begin{array}{c}
w_{1}^{T} \varphi\left(t_{i}\right)+b_{1} \\
\vdots \\
w_{m}^{T} \varphi\left(t_{i}\right)+b_{m}
\end{array}\right]+} \\
& {\left[\begin{array}{c}
g_{1}\left(t_{i}\right) \\
\vdots \\
g_{m}\left(t_{i}\right)
\end{array}\right]+\left[\begin{array}{c}
e_{1}\left(t_{i}\right) \\
\vdots \\
w_{1}^{T} \varphi\left(t_{1}\right)+b_{1} \\
\vdots \\
w_{m}^{T} \varphi\left(t_{1}\right)+b_{m}
\end{array}\right], \text { for } i=2, \ldots, N, } \\
& {\left[\begin{array}{c}
p_{1} \\
\vdots \\
p_{m}
\end{array}\right] . }
\end{aligned}
$$

Lemma 3.1. Given a positive definite kernel function $K$ : $\mathbb{R} \times \mathbb{R} \rightarrow \mathbb{R}$ with $K(t, s)=\varphi(t)^{T} \varphi(s)$ and a regularization constant $\gamma \in \mathbb{R}^{+}$, the solution to (8) is given by the following dual problem:

$\left[\begin{array}{c|c|c}\mathcal{K} & \mathcal{S} & -F_{A} \\ \hline \mathcal{S}^{T} & \Omega_{0}^{0}(1,1) \times I_{m \times m} & I_{m \times m} \\ \hline-F_{A}^{T} & I_{m \times m} & 0_{m \times m}\end{array}\right]\left[\begin{array}{c}\alpha \\ \hline \beta \\ \hline b\end{array}\right]=\left[\begin{array}{c}G \\ \hline P \\ \hline 0\end{array}\right]$

with

$$
\begin{aligned}
& \alpha=\left[\begin{array}{c}
\frac{\alpha^{1}}{\vdots} \\
\hline \alpha^{m}
\end{array}\right]=\left[\alpha_{2}^{1} ; \ldots ; \alpha_{N}^{1} ; \cdots ; \alpha_{2}^{m} ; \ldots ; \alpha_{N}^{m}\right] \in \mathbb{R}^{m(N-1)}, \\
& \beta=\left[\beta_{1} ; \cdots ; \beta_{m}\right], b=\left[b_{1} ; \ldots ; b_{m}\right], P=\left[p_{1} ; \ldots ; p_{m}\right], \\
& G=\left[g_{1}\left(t_{2}\right) ; \cdots ; g_{1}\left(t_{N}\right) ; \cdots ; g_{m}\left(t_{2}\right) ; \cdots ; g_{m}\left(t_{N}\right)\right] \in \mathbb{R}^{m(N-1)} \text {, } \\
& F_{A}=\left[\begin{array}{ccc}
F_{A_{11}} & \ldots & F_{A_{1 m}} \\
\vdots & & \vdots \\
F_{A_{m 1}} & \ldots & F_{A_{m m}}
\end{array}\right] \in \mathbb{R}^{m(N-1) \times m}, \\
& F_{A_{k l}}=\left[a_{k l}\left(t_{2}\right) ; \cdots ; a_{k l}\left(t_{N}\right)\right] \in \mathbb{R}^{N-1}, \text { for } k, l=1, \ldots, m \\
& F_{Z_{k l}}=\left[z_{k l}\left(t_{2}\right) ; \cdots ; z_{k l}\left(t_{N}\right)\right] \in \mathbb{R}^{N-1} \text {, for } k, l=1, \ldots, m \\
& \mathcal{K}=\left[\begin{array}{ccc}
\mathcal{K}_{11} & \ldots & \mathcal{K}_{1 m} \\
\vdots & & \vdots \\
\mathcal{K}_{m 1} & \ldots & \mathcal{K}_{m m}
\end{array}\right] \in \mathbb{R}^{m(N-1) \times m(N-1)}, \\
& \mathcal{K}_{i i}=\overline{D_{Z_{i}}} \bar{\Omega}_{1}^{1}{\overline{D_{Z_{i}}}}^{T}-\overline{D_{A_{i}}} \bar{\Omega}_{1}^{0}{\overline{D_{Z_{i}}}}^{T}-{\overline{D_{Z_{i}}}}_{\Omega_{0}^{1}}^{1}{\overline{D_{A_{i}}}}^{T}+ \\
& \overline{D_{A_{i}}} \bar{\Omega}_{0}^{0}{\overline{D_{A_{i}}}}^{T}+I_{N-1} / \gamma, i=1, \ldots, m, \\
& \mathcal{K}_{i j}=\overline{D_{Z_{i}}} \bar{\Omega}_{1}^{1}{\overline{D_{Z_{j}}}}^{T}-\overline{D_{A_{i}}} \bar{\Omega}_{1}^{0}{\overline{D_{Z_{j}}}}^{T}-\overline{D_{Z_{i}}} \bar{\Omega}_{0}^{1}{\overline{D_{A_{j}}}}^{T}+ \\
& \overline{D_{A_{i}}} \bar{\Omega}_{0}^{0}{\overline{D_{A_{j}}}}^{T}, i, j=1, \ldots, m \text { and } i \neq j \text {, } \\
& \mathcal{S}=\left[\begin{array}{ccc}
\mathcal{S}_{11} & \ldots & \mathcal{S}_{1 m} \\
\vdots & & \vdots \\
\mathcal{S}_{m 1} & \ldots & \mathcal{S}_{m m}
\end{array}\right] \in \mathbb{R}^{m(N-1) \times m}, \\
& \mathcal{S}_{i j}=D_{Z_{i j}} \Omega_{0}^{1}(1,:)^{T}-D_{A_{i j}} \Omega_{0}^{0}(1,:)^{T}, i, j=1, \ldots, m, \\
& \overline{D_{Z_{i}}}=\left[D_{Z_{i 1}}, \ldots, D_{Z_{i m}}\right], \overline{D_{A_{i}}}=\left[D_{A_{i 1}}, \ldots, D_{A_{i m}}\right] \text {, } \\
& \bar{\Omega}_{l}^{k}=\left[\begin{array}{ccc}
\tilde{\Omega}_{k}^{l} & & \\
& \ddots & \\
& & \tilde{\Omega}_{k}^{l}
\end{array}\right] \in \mathbb{R}^{m(N-1) \times m(N-1)}, k, l=0,1 .
\end{aligned}
$$

$D_{A_{i j}}$ and $D_{Z_{i j}}$ are diagonal matrices with the elements of $F_{A_{i j}}$ and $F_{Z_{i j}}$ on the main diagonal respectively. $\Omega_{k}^{l}(1,:)=$ $\left[\Omega_{k}^{l}(1,2) ; \ldots ; \Omega_{k}^{l}(1, N)\right]^{T}$ and $\tilde{\Omega}_{k}^{l}=\Omega_{k}^{l}(2: N, 2: N)$ for $k, l=$ 0,1 . Also note that $\mathcal{K}=\mathcal{K}^{T}$.

Proof. Consider the Lagrangian of problem (8):

$$
\begin{aligned}
& \mathscr{L}\left(w_{\ell}, b_{\ell}, e_{\ell}^{i}, \alpha_{i}^{\ell}, \beta_{\ell}\right)= \\
& \frac{1}{2} \sum_{\ell=1}^{m} w_{\ell}^{T} w_{\ell}+\frac{\gamma}{2} \sum_{\ell=1}^{m} e_{\ell}^{T} e_{\ell}-\sum_{\ell=1}^{m}\left[\sum _ { i = 2 } ^ { N } \alpha _ { i } ^ { \ell } \left[w _ { \ell } ^ { T } \left(z_{\ell \ell}\left(t_{i}\right) \varphi^{\prime}\left(t_{i}\right)-\right.\right.\right. \\
& \left.a_{\ell \ell}\left(t_{i}\right) \varphi\left(t_{i}\right)\right)-\sum_{\substack{k=1 \\
k \neq \ell}}^{m} w_{k}^{T}\left(z_{\ell k}\left(t_{i}\right) \varphi^{\prime}\left(t_{i}\right)-a_{\ell k}\left(t_{i}\right) \varphi\left(t_{i}\right)\right)- \\
& \left.\left.\sum_{k=1}^{m} a_{\ell k}\left(t_{i}\right) b_{k}-e_{\ell}^{i}-g_{\ell}^{i}\right]\right]-\sum_{\ell=1}^{m} \beta_{\ell}\left(w_{\ell}^{T} \varphi\left(t_{1}\right)+b_{\ell}-p_{\ell}\right)
\end{aligned}
$$

where $\left\{\alpha_{i}^{\ell}\right\}_{i=2}^{N}$, for $\ell=1, \ldots, m$ and $\left\{\beta_{\ell}\right\}_{\ell=1}^{m}$ are Lagrange multipliers. $g_{\ell}^{i}=g_{\ell}\left(t_{i}\right), e_{\ell}^{i}=e_{\ell}\left(t_{i}\right)$ for $i=2, \ldots, N$ and $\ell=1, \ldots, m$. 
Then the Karush-Kuhn-Tucker(KKT) optimality conditions are as follows,

$$
\begin{aligned}
& \frac{\partial \mathscr{L}}{\partial w_{\ell}}=0 \rightarrow w_{\ell}=\sum_{v=1}^{m} \sum_{i=2}^{N} \alpha_{i}^{v}\left(z_{v \ell}\left(t_{i}\right) \varphi^{\prime}\left(t_{i}\right)-a_{v \ell}\left(t_{i}\right) \varphi\left(t_{i}\right)\right)+ \\
& \beta_{\ell} \varphi\left(t_{1}\right), \ell=1, \ldots, m, \\
& \frac{\partial \mathscr{L}}{\partial b_{\ell}}=0 \rightarrow \sum_{k=1}^{m} \sum_{i=2}^{N} \alpha_{i}^{k} a_{k \ell}\left(t_{i}\right)-\beta_{\ell}=0, \ell=1, \ldots, m, \\
& \frac{\partial \mathscr{L}}{\partial e_{\ell}^{i}}=0 \rightarrow e_{\ell}^{i}=-\frac{\alpha_{i}^{\ell}}{\gamma}, \quad i=2, \ldots, N, \ell=1, \ldots, m, \\
& \frac{\partial \mathscr{L}}{\partial \alpha_{i}^{\ell}}=0 \rightarrow \sum_{k=1}^{m} w_{k}^{T}\left(z_{\ell k}\left(t_{i}\right) \varphi^{\prime}\left(t_{i}\right)-a_{\ell k}\left(t_{i}\right) \varphi\left(t_{i}\right)\right) \\
& -\sum_{k=1}^{m} a_{\ell k}\left(t_{i}\right) b_{k}-e_{\ell}^{i}=g_{\ell}^{i}, \quad i=2, \ldots, N, \ell=1, \ldots, m \\
& \frac{\partial \mathscr{L}}{\partial \beta_{\ell}}=0 \rightarrow w_{\ell}^{T} \varphi\left(t_{1}\right)+b_{\ell}=p_{\ell}, \ell=1, \ldots, m .
\end{aligned}
$$

Eliminating $\left\{w_{\ell}\right\}_{\ell=1}^{m}$ and $\left\{e_{\ell}^{i}\right\}_{i=2}^{N}$ for $\ell=1, \ldots, m$ from the corresponding KKT optimality conditions yields the following set of equations

$$
\left\{\begin{aligned}
g_{\ell}^{i}= & \sum_{v=1}^{m}\left[\sum _ { j = 2 } ^ { N } \alpha _ { j } ^ { v } \left[\sum_{k=1}^{m} z_{\ell k}\left(t_{i}\right) \Omega_{1}^{1}(j, i) z_{v k}\left(t_{j}\right)-\right.\right. \\
& \sum_{k=1}^{m} a_{\ell k}\left(t_{i}\right) \Omega_{1}^{0}(j, i) z_{v k}\left(t_{j}\right)-\sum_{k=1}^{m} z_{\ell k}\left(t_{i}\right) \Omega_{0}^{1}(j, i) a_{v k}\left(t_{j}\right)+ \\
& \left.\left.\sum_{k=1}^{m} a_{\ell k}\left(t_{i}\right) \Omega_{0}^{0}(j, i) a_{v k}\left(t_{j}\right)\right]\right]_{+} \\
& \sum_{v=1}^{m} \beta_{v}\left(z_{\ell v}\left(t_{i}\right) \Omega_{0}^{1}(1, i)-a_{\ell v}\left(t_{i}\right) \Omega_{0}^{0}(1, i)\right) \\
& +\frac{\alpha_{i}^{\ell}}{\gamma}-\sum_{k=1}^{m} a_{\ell k}\left(t_{i}\right) b_{k}, i=2, \ldots, N, \text { and } \ell=1, \ldots, m, \\
p_{\ell}= & \sum_{v=1}^{m}\left[\sum_{j=2}^{N} \alpha_{j}^{v}\left(z_{v \ell}\left(t_{j}\right) \Omega_{1}^{0}(j, 1)-a_{v \ell}\left(t_{j}\right) \Omega_{0}^{0}(j, 1)\right)\right] \\
& +\beta_{\ell} \Omega_{0}^{0}(1,1)+b_{\ell}, \ell=1, \ldots, m, \\
0= & \sum_{v=1}^{m} \sum_{j=2}^{N} \alpha_{j}^{v} a_{v \ell}\left(t_{j}\right)-\beta_{\ell}, \ell=1, \ldots, m .
\end{aligned}\right.
$$

Finally writing these equations in matrix form will result in the linear system (9).

The model in the dual form becomes

$$
\begin{gathered}
\hat{x}_{\ell}(t)=\sum_{\nu=1}^{m} \sum_{i=2}^{N} \alpha_{i}^{v}\left(z_{v \ell}\left(t_{i}\right)\left[\nabla_{1}^{0} K\right]\left(t_{i}, t\right)-a_{v \ell}\left(t_{i}\right)\left[\nabla_{0}^{0} K\right]\left(t_{i}, t\right)\right)+ \\
\beta_{\ell}\left[\nabla_{0}^{0} K\right]\left(t_{1}, t\right)+b_{\ell}, \quad \ell=1, \ldots, m .
\end{gathered}
$$

\subsection{Explicit ODE System}

Let us consider the system of IVPs (6) with $Z$ as identity matrix as a special case. This results in an ODE system.

Corollary 3.1. Given a positive definite kernel function $K: \mathbb{R} \times$ $\mathbb{R} \rightarrow \mathbb{R}$ with $K(t, s)=\varphi(t)^{T} \varphi(s)$ and a regularization constant $\gamma \in \mathbb{R}^{+}$, the solution to (8) with the matrix $Z=I$, is then given by the following dual problem:

$$
\left[\begin{array}{c|c|c}
\mathcal{K} & \mathcal{S} & -F_{A} \\
\hline \mathcal{S}^{T} & \Omega_{0}^{0}(1,1) \times I_{m \times m} & I_{m \times m} \\
\hline-F_{A}^{T} & I_{m \times m} & 0_{m \times m}
\end{array}\right]\left[\begin{array}{c}
\alpha \\
\hline \beta \\
\hline b
\end{array}\right]=\left[\begin{array}{c}
G \\
\hline P \\
\hline 0
\end{array}\right]
$$

where $\alpha, \beta, b, R, P$ and $F_{A}$ are defined as in Eq. (9). Also with

$$
\begin{aligned}
& F_{A_{k l}}=\left[a_{k l}\left(t_{2}\right) ; \cdots ; a_{k l}\left(t_{N}\right)\right] \in \mathbb{R}^{N-1}, \text { for } k, l=1, \ldots, m \\
& \mathcal{K}=\left[\begin{array}{ccc}
\mathcal{K}_{11} & \ldots & \mathcal{K}_{1 m} \\
\vdots & & \vdots \\
\mathcal{K}_{m 1} & \ldots & \mathcal{K}_{m m}
\end{array}\right] \in \mathbb{R}^{m(N-1) \times m(N-1)}, \\
& \mathcal{K}_{i i}=\tilde{\Omega}_{1}^{1}-D_{i i} \tilde{\Omega}_{1}^{0}-\tilde{\Omega}_{0}^{1} D_{i i}+T_{i i}+I_{N-1} / \gamma, i=1, \ldots, m, \\
& \mathcal{K}_{i j}=-\tilde{\Omega}_{0}^{1} D_{j i}-D_{i j} \tilde{\Omega}_{1}^{0}+T_{i j}, i, j=1, \ldots, m \text { and } i \neq j \text {, } \\
& \mathcal{S}=\left[\begin{array}{ccc}
\mathcal{S}_{11} & \ldots & \mathcal{S}_{1 m} \\
\vdots & & \vdots \\
\mathcal{S}_{m 1} & \ldots & \mathcal{S}_{m m}
\end{array}\right] \in \mathbb{R}^{m(N-1) \times m}, \\
& \mathcal{S}_{i i}=\Omega_{0}^{1}(1,:)^{T}-D_{i i} \Omega_{0}^{0}(1,:)^{T}, i=1, \ldots, m, \\
& \mathcal{S}_{i j}=-D_{i j} \Omega_{0}^{0}(1,:)^{T}, i, j=1, \ldots, m \text { and } i \neq j \text {, } \\
& T_{i j}=\bar{D}_{i} \bar{\Omega} \bar{D}_{j}^{T} \in \mathbb{R}^{(N-1) \times(N-1)} i, j=1, \ldots, m, \\
& \bar{D}_{i}=\left[D_{i 1}, \ldots, D_{i m}\right], \bar{D}_{j}=\left[D_{j 1}, \ldots, D_{j m}\right] \text {, } \\
& \bar{\Omega}=\left[\begin{array}{ccc}
\tilde{\Omega}_{0}^{0} & & \\
& \ddots & \\
& & \tilde{\Omega}_{0}^{0}
\end{array}\right] \in \mathbb{R}^{m(N-1) \times m(N-1)}
\end{aligned}
$$

$D_{i j}$ is a diagonal matrix with the elements of $F_{i j}$ on the main diagonal. $\Omega_{n}^{m}(1,:)=\left[\Omega_{n}^{m}(1,2) ; \ldots ; \Omega_{n}^{m}(1, N)\right]^{T}$ and $\tilde{\Omega}_{n}^{m}=\Omega_{n}^{m}(2$ : $N, 2: N)$ for $n, m=0,1$. Also note that $\mathcal{K}=\mathcal{K}^{T}$.

The model in the dual form becomes

$$
\begin{aligned}
\hat{x}_{\ell}(t)= & \sum_{i=2}^{N} \alpha_{i}^{\ell}\left(\left[\nabla_{1}^{0} K\right]\left(t_{i}, t\right)-a_{\ell \ell}\left(t_{i}\right)\left[\nabla_{0}^{0} K\right]\left(t_{i}, t\right)\right)- \\
& \sum_{\substack{v=1 \\
v \neq \ell}}^{m} \sum_{i=2}^{N} \alpha_{i}^{v}\left(a_{v \ell}\left(t_{i}\right)\left[\nabla_{0}^{0} K\right]\left(t_{i}, t\right)\right)+ \\
& \beta_{\ell}\left[\nabla_{0}^{0} K\right]\left(t_{1}, t\right)+b_{\ell}, \ell=1, \ldots, m
\end{aligned}
$$

where $K$ is the kernel function.

where $K$ is the kernel function. 


\section{Formulation of the method for BVPs in DAEs}

Consider linear time varying boundary value problem in DAEs of the following from

$$
\begin{aligned}
& Z(t) \dot{X}(t)=A(t) X(t)+g(t), t \in\left[t_{i n}, t_{f}\right], \\
& F X\left(t_{i n}\right)+H X\left(t_{f}\right)=X_{0},
\end{aligned}
$$

where matrices $Z(t), A(t)$ and the state vector $X(t)$ are defined as in Eq. (6). $F=\left[f_{i j}\right]$ and $H=\left[h_{i j}\right] \in \mathbb{R}^{m \times m}$. The input is $g(t)$ and $\dot{X}(t)=\frac{d X}{d t}$. $Z(t)$ may be singular on $\left[t_{i n}, t_{f}\right]$ with variable rank and the DAE may have an index that is larger than one. As before we assume that $Z(t), A(t)$ and $g(t)$ are sufficiently smooth and the DAE (11) is solvable. When $Z$ is nonsingular, equation (11) can be converted to equivalent explicit ODE system.

The approximate solution, $\hat{x}_{i}(t)=w_{i}^{T} \varphi(t)+b_{i}$, for $i=1, \ldots, m$ is then obtained by solving the following optimization problem,

$$
\begin{aligned}
& \underset{w_{i}, b_{i}, e_{\ell}^{i}}{\operatorname{minimize}} \frac{1}{2} \sum_{\ell=1}^{m} w_{\ell}^{T} w_{\ell}+\frac{\gamma}{2} \sum_{\ell=1}^{m} e_{\ell}^{T} e_{\ell}+\frac{\zeta}{2} \sum_{\ell=1}^{m} \xi_{\ell}^{2} \\
& \text { subject to }\left[\begin{array}{ccc}
z_{11}(t) & \cdots & z_{1 m}(t) \\
\vdots & \ddots & \vdots \\
z_{m 1}(t) & \cdots & z_{m m}(t)
\end{array}\right]\left[\begin{array}{c}
w_{1}^{T} \varphi^{\prime}\left(t_{i}\right) \\
\vdots \\
w_{m}^{T} \varphi^{\prime}\left(t_{i}\right)
\end{array}\right]= \\
& {\left[\begin{array}{ccc}
a_{11}(t) & \cdots & a_{1 m}(t) \\
\vdots & \ddots & \vdots \\
a_{m 1}(t) & \cdots & a_{m m}(t)
\end{array}\right]\left[\begin{array}{c}
w_{1}^{T} \varphi\left(t_{i}\right)+b_{1} \\
\vdots \\
w_{m}^{T} \varphi\left(t_{i}\right)+b_{m}
\end{array}\right]+} \\
& {\left[\begin{array}{c}
g_{1}\left(t_{i}\right) \\
\vdots \\
g_{m}\left(t_{i}\right)
\end{array}\right]+\left[\begin{array}{c}
e_{1}\left(t_{i}\right) \\
\vdots \\
e_{m}\left(t_{i}\right)
\end{array}\right] \text {, for } i=2, \ldots, N-1,} \\
& {\left[\begin{array}{ccc}
f_{11} & \cdots & f_{1 m} \\
\vdots & \ddots & \vdots \\
f_{m 1} & \cdots & f_{m m}
\end{array}\right]\left[\begin{array}{c}
w_{1}^{T} \varphi\left(t_{1}\right)+b_{1} \\
\vdots \\
w_{m}^{T} \varphi\left(t_{1}\right)+b_{m}
\end{array}\right]+} \\
& {\left[\begin{array}{ccc}
h_{11} & \cdots & h_{1 m} \\
\vdots & \ddots & \vdots \\
h_{m 1} & \cdots & h_{m m}
\end{array}\right]\left[\begin{array}{c}
w_{1}^{T} \varphi\left(t_{N}\right)+b_{1} \\
\vdots \\
w_{m}^{T} \varphi\left(t_{N}\right)+b_{m}
\end{array}\right]=} \\
& {\left[\begin{array}{c}
p_{1} \\
\vdots \\
p_{m}
\end{array}\right]+\left[\begin{array}{c}
\xi_{1} \\
\vdots \\
\xi_{m}
\end{array}\right]}
\end{aligned}
$$

Lemma 4.1. Given a positive definite kernel function $K: \mathbb{R} \times$ $\mathbb{R} \rightarrow \mathbb{R}$ with $K(t, s)=\varphi(t)^{T} \varphi(s)$ and a regularization constant $\gamma, \xi \in \mathbb{R}^{+}$, the solution to (12) is given by the following dual problem:

$$
\left[\begin{array}{c|c|c}
\mathcal{K} & \mathcal{U} & -F_{A} \\
\hline \mathcal{U}^{T} & \Delta & \Pi \\
\hline-F_{A}^{T} & \Pi^{T} & 0_{m \times m}
\end{array}\right]\left[\begin{array}{c}
\alpha \\
\hline \beta \\
\hline b
\end{array}\right]=\left[\begin{array}{c}
G \\
\hline P \\
\hline 0
\end{array}\right]
$$

with

$$
\begin{aligned}
& \alpha=\left[\begin{array}{c}
\frac{\alpha^{1}}{\vdots} \\
\hline \alpha^{m}
\end{array}\right]=\left[\alpha_{2}^{1} ; \ldots ; \alpha_{N-1}^{1} ; \cdots ; \alpha_{2}^{m} ; \ldots ; \alpha_{N-1}^{m}\right] \in \mathbb{R}^{m(N-2)}, \\
& \beta=\left[\beta_{1} ; \cdots ; \beta_{m}\right], b=\left[b_{1} ; \ldots ; b_{m}\right], P=\left[p_{1} ; \ldots ; p_{m}\right], \\
& G=\left[g_{1}\left(t_{2}\right) ; \cdots ; g_{1}\left(t_{N-1}\right) ; \cdots ; g_{m}\left(t_{2}\right) ; \cdots ; g_{m}\left(t_{N-1}\right)\right] \in \mathbb{R}^{m(N-2)} \text {, } \\
& F_{A}=\left[\begin{array}{ccc}
F_{A_{11}} & \ldots & F_{A_{1 m}} \\
\vdots & & \vdots \\
F_{A_{m 1}} & \ldots & F_{A_{m m}}
\end{array}\right] \in \mathbb{R}^{m(N-2) \times m}, \\
& F_{A_{k l}}=\left[a_{k l}\left(t_{2}\right) ; \cdots ; a_{k l}\left(t_{N-1}\right)\right] \in \mathbb{R}^{N-2}, \text { for } k, l=1, \ldots, m \text {, } \\
& F_{Z_{k l}}=\left[z_{k l}\left(t_{2}\right) ; \cdots ; z_{k l}\left(t_{N-1}\right)\right] \in \mathbb{R}^{N-2}, \text { for } k, l=1, \ldots, m, \\
& \Pi=F+H \text {, } \\
& \Delta=\left(F F^{T}\right) \Omega_{0}^{0}(1,1)+\left(F H^{T}+H F^{T}\right) \Omega_{0}^{0}(1, N)+\left(H H^{T}\right) \Omega_{0}^{0}(N, N) \\
& +I_{m} / \zeta \\
& \mathcal{K}=\left[\begin{array}{ccc}
\mathcal{K}_{11} & \ldots & \mathcal{K}_{1 m} \\
\vdots & & \vdots \\
\mathcal{K}_{m 1} & \ldots & \mathcal{K}_{m m}
\end{array}\right] \in \mathbb{R}^{m(N-2) \times m(N-2)}, \\
& \mathcal{K}_{i i}={\overline{D_{Z_{i}}}}_{{ }_{1}^{1}}^{1}{\overline{D_{Z_{i}}}}^{T}-{\overline{D_{A_{i}}}}_{\Omega_{1}^{0}}^{0}{\overline{Z_{Z_{i}}}}^{T}-{\overline{D_{Z_{i}}}}_{\bar{\Omega}_{0}^{1}}^{1}{\overline{D_{A_{i}}}}^{T}+ \\
& \overline{D_{A_{i}}} \bar{\Omega}_{0}^{0}{\overline{D_{A_{i}}}}^{T}+I_{N-1} / \gamma, i=1, \ldots, m,
\end{aligned}
$$

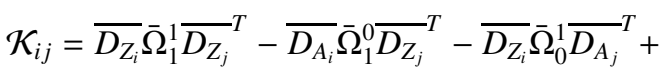

$$
\begin{aligned}
& \overline{D_{A_{i}}} \bar{\Omega}_{0}^{0}{\overline{D_{A_{j}}}}^{T}, i, j=1, \ldots, m \text { and } i \neq j \text {, } \\
& \mathcal{U}=\left[\begin{array}{ccc}
\mathcal{U}_{11} & \ldots & \mathcal{U}_{1 m} \\
\vdots & & \vdots \\
\mathcal{U}_{m 1} & \ldots & \mathcal{U}_{m m}
\end{array}\right] \in \mathbb{R}^{m(N-2) \times m} \\
& \mathcal{U}_{i j}=\left(\sum_{k=1}^{m} D_{Z_{i k}} f_{j k}\right) \Omega_{0}^{1}(1,:)^{T}+\left(\sum_{k=1}^{m} D_{Z_{i k}} h_{j k}\right) \Omega_{0}^{1}(N,:)^{T} \\
& -\left(\sum_{k=1}^{m} D_{A_{i k}} f_{j k}\right) \Omega_{0}^{0}(1,:)^{T}-\left(\sum_{k=1}^{m} D_{A_{i k}} h_{j k}\right) \Omega_{0}^{0}(N,:)^{T}, i, j=1, \ldots, m, \\
& \overline{D_{Z_{i}}}=\left[D_{Z_{i 1}}, \ldots, D_{Z_{i m}}\right], \overline{D_{A_{i}}}=\left[D_{A_{i 1}}, \ldots, D_{A_{i m}}\right] \text {, } \\
& \bar{\Omega}_{l}^{k}=\left[\begin{array}{ccc}
\tilde{\Omega}_{k}^{l} & & \\
& \ddots & \\
& & \tilde{\Omega}_{k}^{l}
\end{array}\right] \in \mathbb{R}^{m(N-2) \times m(N-2)}, k, l=0,1 .
\end{aligned}
$$

$D_{A_{i j}}$ and $D_{Z_{i j}}$ are diagonal matrices with the elements of $F_{A_{i j}}$ and $F_{Z_{i j}}$ on the main diagonal respectively. $\Omega_{k}^{l}(1,:)=$ $\left[\Omega_{k}^{l}(1,2) ; \ldots ; \Omega_{k}^{l}(1, N-1)\right]^{T}, \Omega_{k}^{l}(N,:)=\left[\Omega_{k}^{l}(N, 2) ; \ldots ; \Omega_{k}^{l}(N, N-\right.$ 1)] $]^{T}$ and $\tilde{\Omega}_{k}^{l}=\Omega_{k}^{l}(2: N-1,2: N-1)$ for $k, l=0,1$. Also note that $\mathcal{K}=\mathcal{K}^{T}$.

Proof. Consider the Lagrangian of problem (12): 


$$
\begin{aligned}
& \mathscr{L}\left(w_{\ell}, b_{\ell}, e_{\ell}^{i}, \xi_{\ell}, \alpha_{i}^{\ell}, \beta_{\ell}\right)=\frac{1}{2} \sum_{\ell=1}^{m} w_{\ell}^{T} w_{\ell}+\frac{\gamma}{2} \sum_{\ell=1}^{m} e_{\ell}^{T} e_{\ell}+\frac{\zeta}{2} \sum_{\ell=1}^{m} \xi_{\ell}^{2} \\
& -\sum_{\ell=1}^{m}\left[\sum _ { i = 2 } ^ { N - 1 } \alpha _ { i } ^ { \ell } \left[w_{\ell}^{T}\left(z_{\ell \ell}\left(t_{i}\right) \varphi^{\prime}\left(t_{i}\right)-a_{\ell \ell}\left(t_{i}\right) \varphi\left(t_{i}\right)\right)-\sum_{\substack{k=1 \\
k \neq \ell}}^{m} w_{k}^{T}\left(z_{\ell k}\left(t_{i}\right) \varphi^{\prime}\left(t_{i}\right)-\right.\right.\right. \\
& \left.\left.\left.a_{\ell k}\left(t_{i}\right) \varphi\left(t_{i}\right)\right)-\sum_{k=1}^{m} a_{\ell k}\left(t_{i}\right) b_{k}-e_{\ell}^{i}-g_{\ell}^{i}\right]\right]-\sum_{\ell=1}^{m} \beta_{\ell}\left[\sum _ { i = 1 } ^ { m } w _ { i } ^ { T } \left(f_{\ell i} \varphi\left(t_{1}\right)+\right.\right. \\
& \left.\left.h_{\ell i} \varphi\left(t_{N}\right)\right)+\sum_{i=1}^{m} b_{i}\left(f_{\ell i}+h_{\ell i}\right)-p_{\ell}-\xi_{\ell}\right]
\end{aligned}
$$

where $\left\{\alpha_{i}^{\ell}\right\}_{i=2}^{N-1}$, for $\ell=1, \ldots, m$ and $\left\{\beta_{\ell}\right\}_{\ell=1}^{m}$ are Lagrange multipliers. $g_{\ell}^{i}=g_{\ell}\left(t_{i}\right), e_{\ell}^{i}=e_{\ell}\left(t_{i}\right)$ for $i=2, \ldots, N-1$ and $\ell=1, \ldots, m$. Then the Karush-Kuhn-Tucker (KKT) optimality conditions are as follows,

$$
\begin{aligned}
& \frac{\partial \mathscr{L}}{\partial w_{\ell}}=0 \rightarrow w_{\ell}=\sum_{v=1}^{m} \sum_{i=2}^{N-1} \alpha_{i}^{v}\left(z_{v \ell}\left(t_{i}\right) \varphi^{\prime}\left(t_{i}\right)-a_{v \ell}\left(t_{i}\right) \varphi\left(t_{i}\right)\right)+ \\
& \sum_{j=1}^{m} \beta_{j}\left(f_{j \ell} \varphi\left(t_{1}\right)+h_{j \ell} \varphi\left(t_{N}\right)\right), \ell=1, \ldots, m, \\
& \frac{\partial \mathscr{L}}{\partial b_{\ell}}=0 \rightarrow \sum_{k=1}^{m} \sum_{i=2}^{N-1} \alpha_{i}^{k} a_{k \ell}\left(t_{i}\right)-\sum_{j=1}^{m} \beta_{j}\left(f_{j \ell}+h_{j \ell}\right)=0, \ell=1, \ldots, m, \\
& \frac{\partial \mathscr{L}}{\partial e_{\ell}^{i}}=0 \rightarrow e_{\ell}^{i}=-\frac{\alpha_{i}^{\ell}}{\gamma}, \quad i=2, \ldots, N-1, \ell=1, \ldots, m, \\
& \frac{\partial \mathscr{L}}{\partial \xi_{\ell}}=0 \rightarrow \xi_{\ell}=-\frac{\beta_{\ell}}{\zeta}, \quad \ell=1, \ldots, m, \\
& \frac{\partial \mathscr{L}}{\partial \alpha_{i}^{\ell}}=0 \rightarrow \sum_{k=1}^{m} w_{k}^{T}\left(z_{\ell k}\left(t_{i}\right) \varphi^{\prime}\left(t_{i}\right)-a_{\ell k}\left(t_{i}\right) \varphi\left(t_{i}\right)\right) \\
& -\sum_{k=1}^{m} a_{\ell k}\left(t_{i}\right) b_{k}-e_{\ell}^{i}=g_{\ell}^{i}, \quad i=2, \ldots, N-1, \ell=1, \ldots, m \\
& \frac{\partial \mathscr{L}}{\partial \beta_{\ell}}=0 \rightarrow \sum_{i=1}^{m} w_{i}^{T}\left(f_{\ell i} \varphi\left(t_{1}\right)+h_{\ell i} \varphi\left(t_{N}\right)\right)+\sum_{i=1}^{m} b_{i}\left(f_{\ell i}+h_{\ell i}\right) \\
& \quad-\xi_{\ell}=0, \ell=1, \ldots, m .
\end{aligned}
$$

Eliminating $\left\{w_{\ell}\right\}_{\ell=1}^{m},\left\{e_{\ell}^{i}\right\}_{i=2}^{N-1}$ and $\xi_{\ell}$ for $\ell=1, \ldots, m$ from the corresponding KKT optimality conditions yields the following set of equations:

$$
\begin{aligned}
p_{\ell} & =\sum_{v=1}^{m}\left[\sum _ { j = 2 } ^ { N - 1 } \alpha _ { j } ^ { v } \left[\left(\sum_{k=1}^{m} z_{\ell k}\left(t_{j}\right) f_{v k}\right) \Omega_{1}^{0}(j, 1)+\left(\sum_{k=1}^{m} z_{\ell k}\left(t_{j}\right) h_{v k}\right)\right.\right. \\
& \Omega_{1}^{0}(j, N)-\left(\sum_{k=1}^{m} a_{\ell k}\left(t_{j}\right) f_{v k}\right) \Omega_{0}^{0}(j, 1)-\left(\sum_{k=1}^{m} a_{\ell k}\left(t_{j}\right) h_{v k}\right) \\
& \left.\left.\Omega_{0}^{0}(j, N)\right]\right]+\sum_{j=1}^{m} \beta_{j}\left[\left(\sum_{k=1}^{m} f_{j k} f_{\ell k}\right) \Omega_{0}^{0}(1,1)+\right. \\
& \left.\left(\sum_{k=1}^{m} f_{j k} h_{\ell k}+\sum_{k=1}^{m} h_{j k} f_{\ell k}\right) \Omega_{0}^{0}(1, N)+\left(\sum_{k=1}^{m} h_{j k} h_{\ell k}\right) \Omega_{0}^{0}(N, N)\right] \\
& +\sum_{j=1}^{m} b_{j}\left(f_{\ell j}+h_{\ell j}\right)+\frac{\beta_{\ell}}{\xi}, \ell=1, \ldots, m,
\end{aligned}
$$

$$
\begin{aligned}
g_{\ell}^{i}= & \sum_{v=1}^{m}\left[\sum _ { j = 2 } ^ { N - 1 } \alpha _ { j } ^ { v } \left[\sum_{k=1}^{m} z_{\ell k}\left(t_{i}\right) \Omega_{1}^{1}(j, i) z_{v k}\left(t_{j}\right)-\right.\right. \\
& \sum_{k=1}^{m} a_{\ell k}\left(t_{i}\right) \Omega_{1}^{0}(j, i) z_{v k}\left(t_{j}\right)-\sum_{k=1}^{m} z_{\ell k}\left(t_{i}\right) \Omega_{0}^{1}(j, i) a_{v k}\left(t_{j}\right)+ \\
& \left.\left.\sum_{k=1}^{m} a_{\ell k}\left(t_{i}\right) \Omega_{0}^{0}(j, i) a_{v k}\left(t_{j}\right)\right]\right]+ \\
& \sum_{v=1}^{m} \beta_{v}\left[\left(\sum_{k=1}^{m} z_{\ell k}\left(t_{i}\right) f_{v k}\right) \Omega_{0}^{1}(1, i)+\left(\sum_{k=1}^{m} z_{\ell k}\left(t_{i}\right) h_{v k}\right) \Omega_{0}^{1}(N, i)-\right. \\
& \left.\left(\sum_{k=1}^{m} a_{\ell k}\left(t_{i}\right) f_{v k}\right) \Omega_{0}^{0}(1, i)-\left(\sum_{k=1}^{m} a_{\ell k}\left(t_{i}\right) h_{v k}\right) \Omega_{0}^{0}(N, i)\right] \\
& +\frac{\alpha_{i}^{\ell}}{\gamma}-\sum_{k=1}^{m} a_{\ell k}\left(t_{i}\right) b_{k}, i=2, \ldots, N-1, \text { and } \ell=1, \ldots, m, \\
0= & \sum_{v=1}^{m} \sum_{j=2}^{N-1} \alpha_{j}^{v} a_{v \ell}\left(t_{j}\right)-\sum_{j=1}^{m} b_{j}\left(f_{j \ell}+h_{j \ell}\right), \ell=1, \ldots, m .
\end{aligned}
$$

Finally writing these equations in matrix form will result in the linear system (9).

The model in the dual form becomes

$$
\begin{aligned}
\hat{x}_{\ell}(t)= & \sum_{v=1}^{m} \sum_{i=2}^{N-1} \alpha_{i}^{v}\left(z_{v \ell}\left(t_{i}\right)\left[\nabla_{1}^{0} K\right]\left(t_{i}, t\right)-a_{v \ell}\left(t_{i}\right)\left[\nabla_{0}^{0} K\right]\left(t_{i}, t\right)\right)+ \\
& \sum_{v=1}^{m} \beta_{v}\left(\left[\nabla_{0}^{0} K\right]\left(t_{1}, t\right) f_{v \ell}+\left[\nabla_{0}^{0} K\right]\left(t_{N}, t\right) h_{v \ell}\right)+b_{\ell}, \ell=1, \ldots, m .
\end{aligned}
$$

where $K$ is the kernel function.

\section{Parameter tuning}

The performance of the LS-SVM model depends on the choice of the tuning parameters. In this paper for all experiments the Gaussian RBF kernel is used. Therefore a model is determined by the regularization parameter $\gamma$ and the kernel bandwidth $\sigma$.

It should be noted that as opposed to the regression case, we don't have the target values and we don't have noise. Therefore a quite large value can be taken for the regularization constant $\gamma$, so that the optimization problem (7) is sharply minimized, compared to the noisy case. In this work, the optimal values for $\gamma$ and $\sigma$ are obtained by evaluating the performance of the model on a validation set using a meaningful grid of possible $(\gamma, \sigma)$ combinations. The validation set is defined to be the set of midpoints $V \equiv\left\{v_{i}=\frac{\left(t_{i}+t_{i+1}\right)}{2}, i=1, \ldots, N-1\right\}$. The values that minimize the mean squared error (MSE) on this validation set are then selected.

\section{Numerical Results}

In this section, four experiments are performed to demonstrate the capability of the proposed method for solving initial 
and boundary value problem in DAEs. The accuracy of an approximate solution is measured by means of mean square error (MSE) which is defined as follows:

$$
\mathrm{MSE}_{\text {test }}=\frac{\sum_{i=1}^{M}\left(x\left(t_{i}\right)-\hat{x}\left(t_{i}\right)\right)^{2}}{M}
$$

where $M$ is the number test points. In all the experiments $M$ is set to 200 points on the given domain.

Problem 5.1: Consider the following nonsingular system of time varying ordinary differential equations

$$
\left[\begin{array}{c}
\dot{x}_{1}(t) \\
\dot{x}_{2}(t)
\end{array}\right]=\left[\begin{array}{cc}
0 & 1 \\
\frac{-2}{t^{2}} & \frac{2}{t^{2}}
\end{array}\right]\left[\begin{array}{c}
x_{1}(t) \\
x_{2}(t)
\end{array}\right]+\left[\begin{array}{c}
0 \\
t \log (t)
\end{array}\right]
$$

subject to

$$
x_{1}(1)=1, x_{2}(1)=0 \text {. }
$$

This problem is solved for $t \in[1,10]$ and the approximate solution obtained by the proposed method is compared with the solution obtained by MATLAB built-in solver ode45 in Fig 1. The obtained results with different number of training points are tabulated in Table 1. Note that the subroutine DSolve of Mathematica 6.0 failed to find the analytical solution for the above equation.

Table 1: Numerical results of the proposed method for solving Problem 5.1 on time interval [1,10], with $N$ number of collocation points.

\begin{tabular}{ccc}
\hline & \multicolumn{2}{c}{ MSE $_{\text {test }}$} \\
\cline { 2 - 3 }$N$ & $x_{1}$ & $x_{2}$ \\
\hline 20 & $3.1 \times 10^{-2}$ & $1.2 \times 10^{-3}$ \\
40 & $3.9 \times 10^{-5}$ & $1.4 \times 10^{-6}$ \\
60 & $2.6 \times 10^{-7}$ & $1.1 \times 10^{-8}$ \\
80 & $4.8 \times 10^{-9}$ & $3.5 \times 10^{-10}$ \\
\hline
\end{tabular}
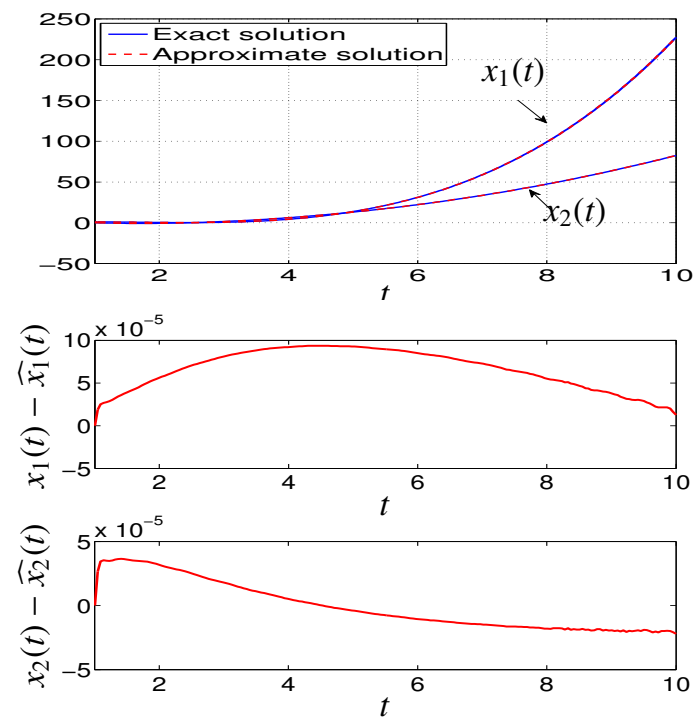

Figure 1: Obtained approximate solution and model errors for problem 5.1, when 80 equidistant mesh points on the interval $[1,10]$ are used for training.

Problem 5.2: Consider the singular system of index-3 discussed as follows [17]:

$$
Z(t) \dot{X}(t)=A(t) X(t)+B(t) u(t), t \in[0,20], X(0)=X_{0}
$$

where

$$
Z=\left[\begin{array}{ccc}
0 & -t & 0 \\
1 & 0 & t \\
0 & 1 & 0
\end{array}\right], A=\left[\begin{array}{ccc}
-1 & 0 & 0 \\
0 & -1 & 0 \\
0 & 0 & -1
\end{array}\right]
$$

and $B(t)=0$ with $x(0)=\left[0, e^{-1}, e^{-1}\right]^{T}$. The exact solution is given by

$$
\begin{aligned}
& x_{1}(t)=-t \exp (-(t+1)), \\
& x_{2}(t)=x_{3}(t)=\exp (-(t+1)) .
\end{aligned}
$$

The problem is solved on domain $t \in[0,20]$ for different $N$ (number of collocation points) values. The approximate solution obtained by the proposed method is compared with the exact solution (see Fig 2) and the results are recorded in Table 2. From Table 2, it is apparent that as $N$ increases, the solution converges to the true solution. Note that the MATLAB built-in solver ode15i can solve DAEs Up to index-1.

Table 2: Numerical results of the proposed method for solving Problem 5.2 on time interval $[0,20]$, with $N$ number of collocation points.

\begin{tabular}{cccc}
\hline & \multicolumn{3}{c}{ MSE $_{\text {test }}$} \\
\cline { 2 - 4 }$N$ & $x_{1}$ & $x_{2}$ & $x_{3}$ \\
\hline 20 & $1.33 \times 10^{-5}$ & $4.82 \times 10^{-8}$ & $4.73 \times 10^{-7}$ \\
40 & $1.38 \times 10^{-8}$ & $1.39 \times 10^{-10}$ & $3.14 \times 10^{-9}$ \\
60 & $4.82 \times 10^{-10}$ & $3.54 \times 10^{-12}$ & $2.38 \times 10^{-10}$ \\
\hline
\end{tabular}
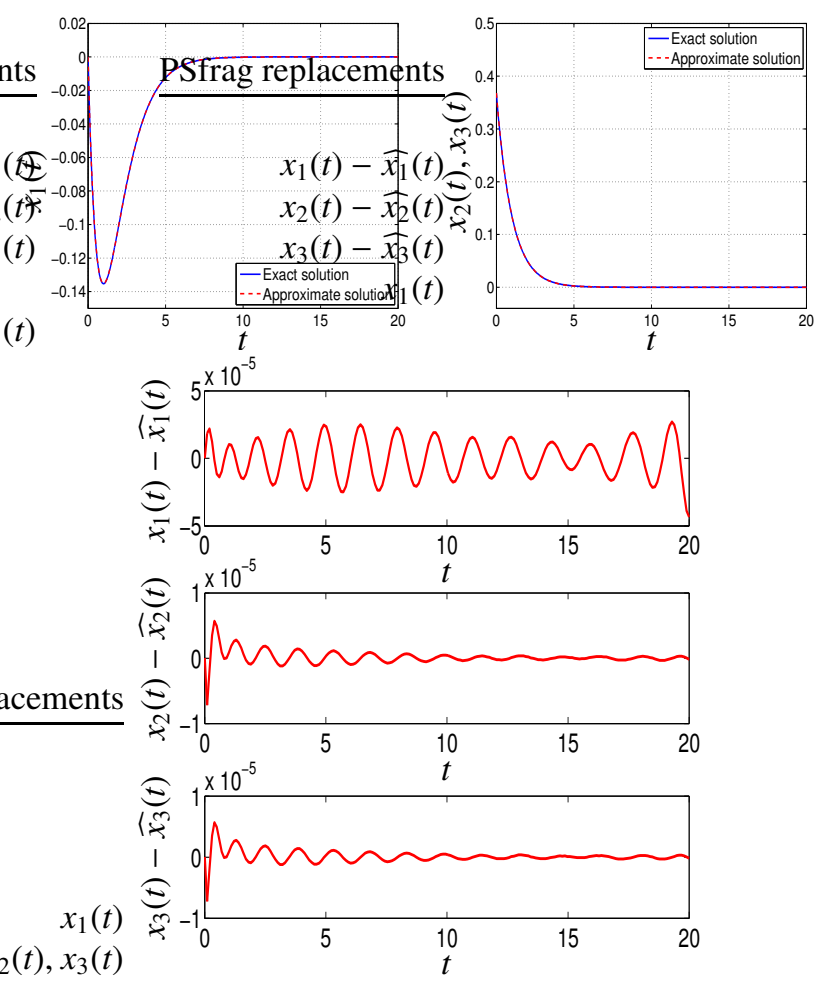

Figure 2: Obtained approximate solution and model errors for problem 5.2, when 70 equidistant mesh points on the interval $[0,20]$ are used for training. 
Problem 5.3: Consider the linear time varying singular system [18]

$$
\begin{aligned}
Z(t) \dot{X}(t) & =A(t) X(t)+B(t) u(t), t \in[0,10], X(0)=X_{0} \\
y(t) & =K(t) x(t)
\end{aligned}
$$

where $y(t)$ is the output vector and

$$
\begin{aligned}
& Z=\left[\begin{array}{cccc}
1+t & 0 & 0 & 0 \\
0 & 0 & 0 & 0 \\
0 & 0 & 1+t & 0 \\
0 & 0 & 0 & 0
\end{array}\right], A=\left[\begin{array}{cccc}
0 & 1 & 0 & 0 \\
1 & 1 & 0 & 0 \\
0 & 0 & 0 & 1 \\
0 & -(1+t) & 1 & 1
\end{array}\right] \\
& B=\left[\begin{array}{ll}
0 & 0 \\
1 & 0 \\
0 & 0 \\
0 & 1
\end{array}\right], K=\left[\begin{array}{cccc}
0 & 1+t & 0 & -1 \\
0 & 0 & 0 & \left(1+e^{-t}\right) \sin (t)
\end{array}\right]
\end{aligned}
$$

with $u=\left[1+t+t^{2} / 2,0\right]^{T}$ and $x(0)=[0,-1,0,-1]^{T}$ the exact solution is given by

$$
\begin{aligned}
& y_{1}(t)=-\left(1+t+t^{2}+\frac{t^{3}}{3}\right)+\frac{1+t+3 t^{2} / 2+t^{3}+t^{4} / 4}{1+t}, \\
& y_{2}(t)=-\left(1+e^{-t}\right) \sin (t)\left(\frac{1+t+3 t^{2} / 2+t^{3}+t^{4} / 4}{1+t}\right) .
\end{aligned}
$$

The interval $[0,10]$ is discretized into $N$ number of mesh points. The obtained mean square errors for test set are tabulated in Table 3. The results reveal that higher order accuracy can be achieved by an increasing number of collocation points. Analytical solution and obtained approximate solution, with 50 equidistant mesh points on the interval $[0,10]$ as training points, are compared in Fig 3. Note that the MATLAB built-in solver ode15i failed to solve problem 5.3.

Table 3: Numerical results of the proposed method for solving Problem 5.3 on time interval $[0,10]$, with $N$ number of collocation points.

\begin{tabular}{ccc}
\hline & \multicolumn{2}{c}{$\mathbf{M S E}_{\text {test }}$} \\
\cline { 2 - 3 }$N$ & $y_{1}$ & $y_{2}$ \\
\hline 10 & $1.23 \times 10^{-3}$ & $8.76 \times 10^{-4}$ \\
20 & $1.25 \times 10^{-5}$ & $1.31 \times 10^{-5}$ \\
30 & $1.42 \times 10^{-6}$ & $7.80 \times 10^{-7}$ \\
40 & $5.35 \times 10^{-9}$ & $7.62 \times 10^{-9}$ \\
\hline
\end{tabular}

Problem 5.4: Consider the linear time varying index one boundary value problem of DAE given by [9]:

$$
Z(t) \dot{X}(t)=A(t) X(t)+g(t), t \in[0,1]
$$

where

$$
Z=\left[\begin{array}{ccc}
1 & -t & t^{2} \\
0 & 1 & -t \\
0 & 0 & 0
\end{array}\right], A=\left[\begin{array}{ccc}
-1 & (t+1) & -\left(t^{2}+2 t\right) \\
0 & 1 & 1-t \\
0 & 0 & -1
\end{array}\right]
$$

with $g(t)=[0,0, \sin (t)]^{T}$ and boundary conditions

$$
x_{1}(0)=1, x_{2}(1)-x_{3}(1)=e .
$$
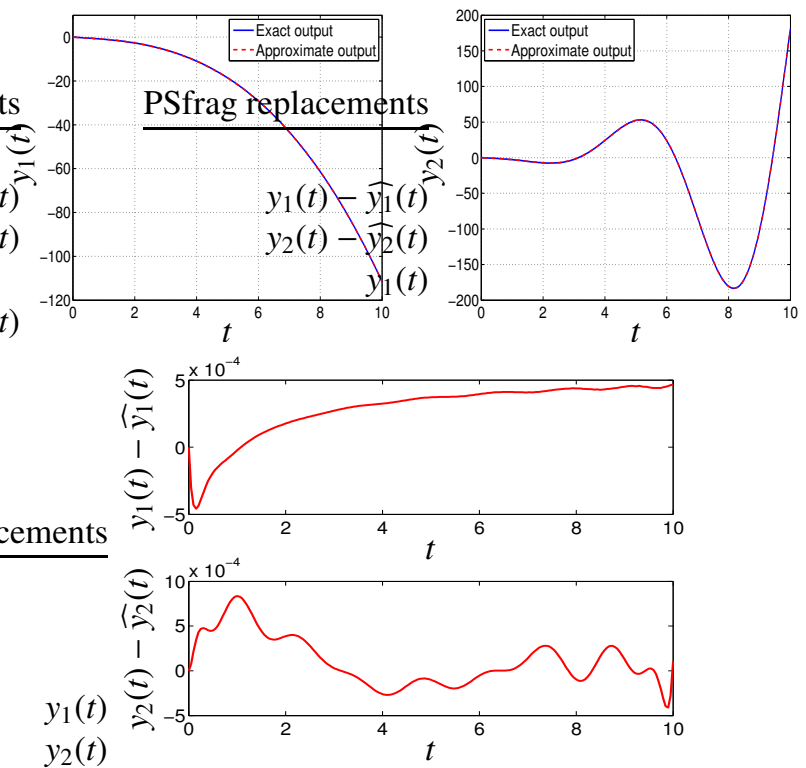

Figure 3: Obtained approximate solution and model errors for problem 5.3, when 50 equidistant mesh points on the interval $[0,10]$ are used for training.

The exact solution is given by

$$
\begin{aligned}
& x_{1}(t)=e^{-t}+t e^{t}, \\
& x_{2}(t)=e^{t}+t \sin (t), \\
& x_{3}(t)=\sin (t) .
\end{aligned}
$$

The computed residuals are displayed in Fig 4. The mean square errors for the test set are also recorded in Table 4.

Table 4: Numerical results of the proposed method for solving Problem 5.4 on time interval $[0,1]$, with $N$ number of collocation points.

\begin{tabular}{cccc}
\hline & \multicolumn{3}{c}{ MSE $_{\text {test }}$} \\
\cline { 2 - 4 }$N$ & $x_{1}$ & $x_{2}$ & $x_{3}$ \\
\hline 10 & $3.81 \times 10^{-5}$ & $2.65 \times 10^{-5}$ & $6.02 \times 10^{-6}$ \\
20 & $2.60 \times 10^{-13}$ & $1.05 \times 10^{-12}$ & $1.65 \times 10^{-12}$ \\
30 & $9.77 \times 10^{-15}$ & $2.78 \times 10^{-13}$ & $3.74 \times 10^{-13}$ \\
40 & $1.04 \times 10^{-15}$ & $7.43 \times 10^{-15}$ & $6.12 \times 10^{-15}$ \\
\hline
\end{tabular}

Remark 2. A singular system with a discontinuous input will exhibit a jump. The LS-SVM approximation with Gaussian kernel (which provides a smooth approximation) shows a spurious oscillation near the discontinuity. This oscillation behavior is a common phenomenon known as the Gibbs phenomenon that appears when the underlying function being approximated has jump discontinuities. Some methods have been suggested in the literature to reduce the effect of Gibbs phenomenon (see [13]). Another approach is to use a continuous approximation of the non smooth input signal as the new input for the system [20].

Remark 3. Concerning practical application of the proposed method for finding the approximate solution to the given DAEs on a very long time interval, the approach described in [16] which is based on the domain decomposition technique can be utilized here as well. 

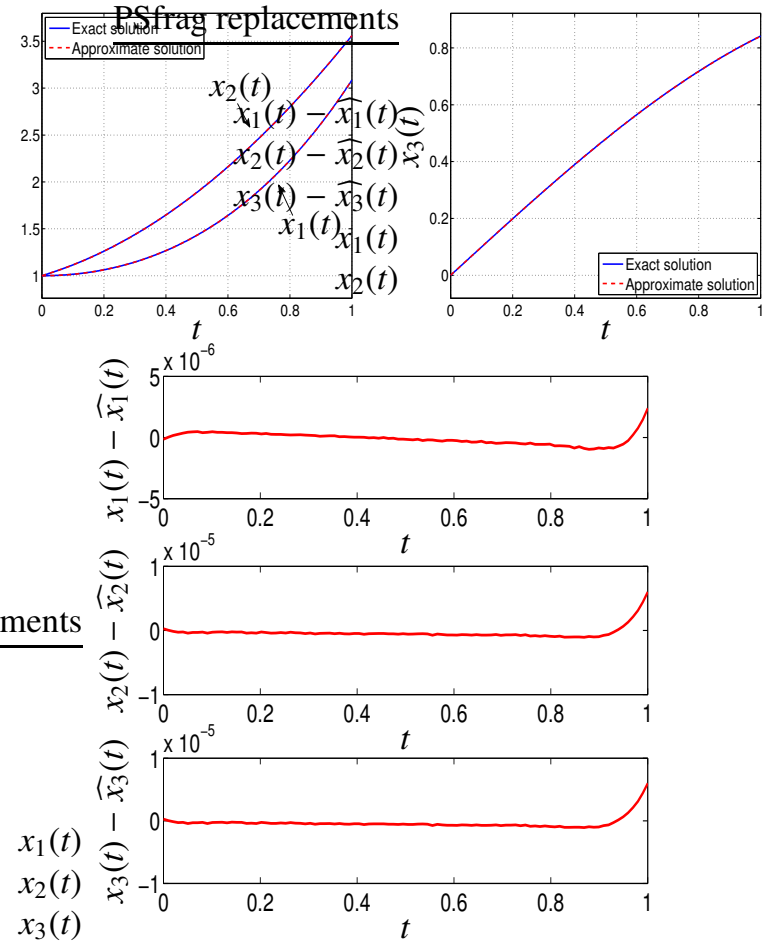

Figure 4: Obtained approximate solution and model errors for problem 5.4, when 10 equidistant mesh points on the interval $[0,1]$ are used for training.

Remark 4. In general, but not necessarily, one starts with equidistant mesh points. The residual error on the validation set is then monitored and for the subintervals which the error is not sufficiently small one may refine the mesh in order to achieve adequate accuracy. The residual error at a given point $t_{i}$ can be computed as

$$
e\left(t_{i}\right)=Z\left(t_{i}\right) \dot{X}\left(t_{i}\right)-A\left(t_{i}\right) X\left(t_{i}\right)-g\left(t_{i}\right)
$$

\section{Conclusion and future work}

In this paper, a new method based on least squares support vector machines is developed for solving linear time varying differential algebraic equations. The method is successfully applied for solving higher index DAEs (index larger than one). The results reveal that the method can obtain the desired accuracy with only a few number of collocation points. Also the method is stable when the non uniform sampling is used to create the collocation points.

\section{Acknowledgments}

This work was supported by Research Council KUL: GOA/11/05 Ambiorics, GOA/10/09 MaNet, CoE EF/05/006 Optimization in Engineering(OPTEC), IOFSCORES4CHEM, several $\mathrm{PhD} /$ postdoc \& fellow grants;Flemish Government:FWO: $\mathrm{PhD} /$ postdoc grants, projects: G0226.06 (cooperative systems and optimization), G0321.06 (Tensors), G.0302.07 (SVM/Kernel), G.0320.08 (convex MPC), G.0558.08 (Robust MHE), G.0557.08 (Glycemia2), G.0588.09 (Brain-machine), G.0377.12 (structured models) research communities (WOG: ICCoS, ANMMM, MLDM); G.0377.09 (Mechatronics MPC) IWT: PhD Grants, Eureka-Flite+, SBO LeCoPro, SBO Climaqs, SBO POM, O\&O-Dsquare; Belgian Federal Science Policy Office: IUAP P6/04 (DYSCO, Dynamical systems, control and optimization, 2007-2011); EU: ERNSI; FP7-HD-MPC (INFSO-ICT-223854), COST intelliCIS, FP7-EMBOCON (ICT-248940); Contract Research: AMINAL; Other:Helmholtz: viCERP, ACCM, Bauknecht, Hoerbiger, ERC AdG A-DATADRIVE-B. Johan Suykens is a professor at the KU Leuven, Belgium.

\section{References}

[1] Ascher, U.M. (1989). On symmetric schemes and differential-algebraic equations, SIAM J. Sci. Stat. Comput. 10 937-949.

[2] Ascher, U.M., Petzold, L.R. (1998). Computer Methods for Ordinary Differential Equations and Differential Algebraic Equations, Society for Industrial and Applied Mathematics, Philadelphia.

[3] Ascher, U.M., Spiter, R.J. (1994). Collocation software for boundaryvalue differential algebraic equations, SIAM J. Sci. Comput. 15, 938-952.

[4] Ascher, U.M., Petzold, L.R. (1991). Projected implicit Runge-Kutta methods for differential algebraic equations, SIAM J. Numer. Anal. 28, 1097-1120.

[5] Awawdeh, F., Jaradat, H.M., \& Alsayyed, O. (2009). Solving system of DAEs by homotopy analysis method, Chaos, Solitons \& Fractals, 42(3), 1422-1427.

[6] Bernan, K. (1986). Numerical simulation of trajectory prescribed path control problems, IEEE Trans. Automat. Control, AC-31, pp. 266-269.

[7] Brenan, K.E., Campbell, S.L., Petzold, L.R. (1989). Numerical Solution of Initial-Value Problems in Differential-Algebraic Equations, Elsevier, New York.

[8] Campbell, S.L. (1982). Singular systems of differential equations II, Pitman, Marshfield, MA.

[9] Clark, K.D., \& Petzold, L.R. (1989). Numerical Solution of Boundary Value Problems in Differential-Algebraic Systems, SIAM J. SCI. STAT. COMPUT, 10(5), 915-936.

[10] Froment, G.B., \& Bischoff, K.B. (1990). Chemical reactor analysis and design. John Wiley \& Sons Inc., New York.

[11] Gear, C.W., Petzold, L.R. (1984). ODE systems for the solution of differential-algebraic systems, SIAM J. Numer. Anal. 21 ,716-728.

[12] Guzel, N., Bayram, M. (2006). Numerical solution of differentialalgebraic equations with index-2, Applied Mathematics and Computation, $174,1279-1289$.

[13] Jerri, A.J. (2011), Advances in the Gibbs Phenomenon, first ed., Sampling Publishing, New York.

[14] Kincaid, D.R., Cheney, E.W. (2002). Numerical Analysis: Mathematics of Scientific Computing, third ed., Brooks/Cole, Pacific Grove, CA.

[15] Lötstedt, P., \& Petzold, L. (1986). Numerical solution of nonlinear differential equations with algebraic constraints: Convergence results for the backward differentiation formulas, Math. Comp., 46, pp. 491-516.

[16] Mehrkanoon, S., Falck, T., \& Suykens, J.A.K. (2011). Approximate Solutions to Ordinary Differential Equations Using Least Squares Support Vector Machines, accepted for publication in IEEE Transactions on Neural Networks and Learning Systems.

[17] Murugesan, K., \& Balakumar, V. (2011). Study on singular systems of index three via runge-kutta method of order-10, IJPAM: 70(5), 723-733.

[18] Murugesan, K., Dhayabaran, D.P., \& Amirtharaj, E.C.H (2002). A study of second-order state-space systems of time-invariant and time-varying transistor circuits using the STWS technique, International Journal of Electronics 89(4), 305-315.

[19] Murugesh, V., Murugesan, K. (2009). RK-Butcher algorithms for singular system-based electronic circuit, International Journal of Computer Mathematics, 86(3), 523-536.

[20] Rabier, P.J., Rheinboldt, W.C. (1996). Time-Dependent Linear DAEs With Discontinuous Inputs, Linear Algebra and its Applications, 247(1), 1-29.

[21] Pengfei L, Yaoyu L \& Seem, J.E. (2012). Consistent initialization of system of differentialalgebraic equations for dynamic simulation of centrifugal chillers, Journal of Building Performance Simulation, 5(2), 115-139.

[22] Ranade, V.V. (2002). Computational fow modeling for chemical engineering. Academic Press, San Diego.

[23] Suykens, J.A.K., Van Gestel, T., De Brabanter, J., De Moor, B., Vandewalle, J. (2002). Least Squares Support Vector Machines, World Scientific Pub. Co., Singapore.

[24] Vapnik, V. (1998). Statistical learning theory, New York: Wiley.

[25] Wang, H.S., Jhu, W.L., Yung, C.F., Wang, P.F. (2011). Numerical Solutions of Differential-Algebraic Equations and Its Applications in Solving TPPC Problems, Journal of Marine Science and Technology, 19(1), 7688. 